\title{
A Counter-Example to the Mismatched Decoding Converse for Binary-Input Discrete Memoryless Channels
}

Jonathan Scarlett, Anelia Somekh-Baruch, Alfonso Martinez and Albert Guillén i Fàbregas

\begin{abstract}
This paper studies the mismatched decoding problem for binary-input discrete memoryless channels. An example is provided for which an achievable rate based on superposition coding exceeds the LM rate (Hui, 1983; CsiszárKörner, 1981), thus providing a counter-example to a previously reported converse result (Balakirsky, 1995). Both numerical evaluations and theoretical results are used in establishing this claim.
\end{abstract}

\section{INTRODUCTION}

In this paper, we consider the problem of channel coding with a given (possibly suboptimal) decoding rule, i.e. mismatched decoding [1]-[4]. This problem is of significant interest in settings where the optimal decoder is ruled out due to channel uncertainty or implementation constraints, and also has several connections to theoretical problems such as zero-error capacity. Finding a single-letter expression for the channel capacity with mismatched decoding is a long-standing open problem, and is believed to be very difficult; the vast majority of the literature has focused on achievability results. The only reported single-letter converse result for general decoding metrics is that of Balakirsky [5], who considered binary-input discrete memoryless channels (DMCs) and stated a matching

J. Scarlett is with the Laboratory for Information and Inference Systems, École Polytechnique Fédérale de Lausanne, CH-1015, Switzerland (e-mail: jmscarlett@gmail.com).

A. Somekh-Baruch is with the Faculty of Engineering at Bar-Ilan University, Ramat-Gan, Israel. (e-mail: somekha@biu.ac.il).

A. Martinez is with the Department of Information and Communication Technologies, Universitat Pompeu Fabra, 08018 Barcelona, Spain (e-mail: alfonso.martinez@ieee.org).

A. Guillén i Fàbregas is with the Institució Catalana de Recerca i Estudis Avançats (ICREA), the Department of Information and Communication Technologies, Universitat Pompeu Fabra, 08018 Barcelona, Spain, and also with the Department of Engineering, University of Cambridge, Cambridge, CB2 1PZ, U.K. (e-mail: guillen@ieee.org).

This work has been funded by the European Research Council under ERC grant agreement 259663, by the European Union's 7th Framework Programme under grant agreement 303633, by the Spanish Ministry of Economy and Competitiveness under grants RYC-2011-08150 and TEC2012-38800-C03-03, and by the Israel Science Foundation under Grant 2013/919.

This work was presented at the Information Theory and Applications Workshop (2015), and is an extended version of a paper accepted to the IEEE Transactions on Information Theory. 
converse to the achievable rate of Hui [1] and Csiszár-Körner [2]. However, in the present paper, we provide a counter-example to this converse, i.e. a binary-input DMC for which this rate can be exceeded.

We proceed by describing the problem setup. The encoder and decoder share a codebook $\mathcal{C}=\left\{\boldsymbol{x}^{(1)}, \ldots \boldsymbol{x}^{(M)}\right\}$ containing $M$ codewords of length $n$. The encoder receives a message $m$ equiprobable on the set $\{1, \ldots M\}$ and transmits $\boldsymbol{x}^{(m)}$. The output sequence $\boldsymbol{y}$ is generated according to $W^{n}(\boldsymbol{y} \mid \boldsymbol{x})=\prod_{i=1}^{n} W\left(y_{i} \mid x_{i}\right)$, where $W$ is a single-letter transition law from $\mathcal{X}$ to $\mathcal{Y}$. The alphabets are assumed to be finite, and hence the channel is a DMC. Given the output sequence $\boldsymbol{y}$, an estimate of the message is formed as follows:

$$
\hat{m}=\arg \max _{j} q^{n}\left(\boldsymbol{x}^{(j)}, \boldsymbol{y}\right),
$$

where $q^{n}(\boldsymbol{x}, \boldsymbol{y}) \triangleq \prod_{i=1}^{n} q\left(x_{i}, y_{i}\right)$ for some non-negative function $q$ called the decoding metric. An error is said to have occurred if $\hat{m}$ differs from $m$, and the error probability is denoted by

$$
p_{\mathrm{e}} \triangleq \mathbb{P}[\hat{m} \neq m]
$$

We assume that ties are broken as errors. A rate $R$ is said to be achievable if, for all $\delta>0$, there exists a sequence of codebooks with $M \geq e^{n(R-\delta)}$ codewords having vanishing error probability under the decoding rule in (1). The mismatched capacity of $(W, q)$ is defined to be the supremum of all achievable rates, and is denoted by $C_{\mathrm{M}}$.

In this paper, we focus on binary-input DMCs, and we will be primarily interested in the achievable rates based on constant-composition codes due to Hui [1] and Csiszár and Körner [2], an achievable rate based on superposition coding by the present authors [6]-[8], and a reported converse by Balakirsky [5]. These are introduced in Sections I-B and I-C.

\section{A. Notation}

The set of all probability mass functions (PMFs) on a given finite alphabet, say $\mathcal{X}$, is denoted by $\mathcal{P}(\mathcal{X})$, and similarly for conditional distributions (e.g. $\mathcal{P}(\mathcal{Y} \mid \mathcal{X})$ ). The marginals of a joint distribution $P_{X Y}(x, y)$ are denoted by $P_{X}(x)$ and $P_{Y}(y)$. Similarly, $P_{Y \mid X}(y \mid x)$ denotes the conditional distribution induced by $P_{X Y}(x, y)$. We write $P_{X}=\widetilde{P}_{X}$ to denote element-wise equality between two probability distributions on the same alphabet. Expectation with respect to a distribution $P_{X}(x)$ is denoted by $\mathbb{E}_{P}[\cdot]$. Given a distribution $Q(x)$ and a conditional distribution $W(y \mid x)$, the joint distribution $Q(x) W(y \mid x)$ is denoted by $Q \times W$. Information-theoretic quantities with respect to a given distribution (e.g. $\left.P_{X Y}(x, y)\right)$ are written using a subscript (e.g. $I_{P}(X ; Y)$ ). All logarithms have base $e$, and all rates are in nats/use.

\section{B. Achievability}

The most well-known achievable rate in the literature, and the one of the most interest in this paper, is the LM rate, which is given as follows for an arbitrary input distribution $Q \in \mathcal{P}(\mathcal{X})$ :

$$
I_{\mathrm{LM}}(Q) \triangleq \min _{\substack{\widetilde{P}_{X Y} \in \mathcal{P}(\mathcal{X} \times \mathcal{Y}): \widetilde{P}_{X}=Q, \widetilde{P}_{Y}=P_{Y} \\ \mathbb{E}_{\tilde{P}}[\log q(X, Y)] \geq \mathbb{E}_{P}[\log q(X, Y)]}} I_{\widetilde{P}}(X ; Y),
$$


where $P_{X Y} \triangleq Q \times W$. This rate was derived independently by Hui [1] and Csiszár-Körner [2]. The proof uses a standard random coding construction in which each codeword is independently drawn according to the uniform distribution on a given type class. The following alternative expression was given by Merhav et al. [4] using Lagrange duality:

$$
I_{\mathrm{LM}}(Q) \triangleq \sup _{s \geq 0, a(\cdot)} \sum_{x, y} Q(x) W(y \mid x) \log \frac{q(x, y)^{s} e^{a(x)}}{\sum_{\bar{x}} Q(\bar{x}) q(\bar{x}, y)^{s} e^{a(\bar{x})}}
$$

Since the input distribution $Q$ is arbitrary, we can optimize it to obtain the achievable rate $C_{\mathrm{LM}} \triangleq \max _{Q} I_{\mathrm{LM}}(Q)$. In general, $C_{\mathrm{M}}$ may be strictly higher than $C_{\mathrm{LM}}[2]$, [9].

The first approach to obtaining achievable rates exceeding $C_{\mathrm{LM}}$ was given in [2]. The idea is to code over pairs of symbols: If a rate $R$ is achievable for the channel $W^{(2)}\left(\left(y_{1}, y_{2}\right) \mid\left(x_{1}, x_{2}\right)\right) \triangleq W\left(y_{1} \mid x_{1}\right) W\left(y_{2} \mid x_{2}\right)$ with the metric $q^{(2)}\left(\left(x_{1}, x_{2}\right),\left(y_{1}, y_{2}\right)\right) \triangleq q\left(x_{1}, y_{1}\right) q\left(x_{2}, y_{2}\right)$, then $\frac{R}{2}$ is achievable for the original channel $W$ with the metric $q$. Thus, one can apply the LM rate to $\left(W^{(2)}, q^{(2)}\right)$, optimize the input distribution on the product alphabet, and infer an achievable rate for $(W, q)$; we denote this rate by $C_{\mathrm{LM}}^{(2)}$. An example was given in [2] for which $C_{\mathrm{LM}}^{(2)}>C_{\mathrm{LM}}$. Moreover, as stated in [2], the preceding arguments can be applied to the $k$-th order product channel for $k>2$; we denote the corresponding achievable rate by $C_{\mathrm{LM}}^{(k)}$. It was conjectured in [2] that $\lim _{k \rightarrow \infty} C_{\mathrm{LM}}^{(k)}=C_{\mathrm{M}}$. It should be noted that the computation of $C_{\mathrm{LM}}^{(k)}$ is generally prohibitively complex even for relatively small values of $k$, since $I_{\mathrm{LM}}(Q)$ is non-concave in general [10].

Another approach to improving on $C_{\mathrm{LM}}$ is to use multi-user random coding ensembles exhibiting more structure than the standard ensemble containing independent codewords. This idea was first proposed by Lapidoth [9], who used parallel coding techniques to provide an example where $C_{\mathrm{M}}=C$ (with $C$ being the matched capacity) but $C_{\mathrm{LM}}<C$. Building on these ideas, further achievable rates were provided by the present authors [6]-[8] using superposition coding techniques. Of particular interest in this paper is the following. For any finite auxiliary alphabet $\mathcal{U}$ and input distribution $Q_{U X}$, the rate $R=R_{0}+R_{1}$ is achievable for any $\left(R_{0}, R_{1}\right)$ satisfying 1

$$
\begin{gathered}
R_{1} \leq \min _{\substack{\widetilde{P}_{U X Y} \in \mathcal{P}(\mathcal{U} \times \mathcal{X} \times \mathcal{Y}): \widetilde{P}_{U X}=Q_{U X}, \widetilde{P}_{U Y}=P_{U Y} \\
\mathbb{E}_{\widetilde{P}}[\log q(X, Y)] \geq \mathbb{E}_{P}[\log q(X, Y)]}} I_{\widetilde{P}}(X ; Y \mid U) \\
R_{0} \leq \min _{\substack{\widetilde{P}_{U X Y} \in \mathcal{P}(\mathcal{U} \times \mathcal{X} \times \mathcal{Y}): \widetilde{P}_{U X}=Q_{U X}, \widetilde{P}_{Y}=P_{Y} \\
\mathbb{E}_{\widetilde{P}}[\log q(X, Y)] \geq \mathbb{E}_{P}[\log q(X, Y)]}} I_{\widetilde{P}}(U ; X)+\left[I_{\widetilde{P}}(X ; Y \mid U)-R_{1}\right]^{+},
\end{gathered}
$$

where $P_{U X Y} \triangleq Q_{U X} \times W$. We define $I_{\mathrm{SC}}\left(Q_{U X}\right)$ to be the maximum of $R_{0}+R_{1}$ subject to these constraints, and we write the optimized rate as $C_{\mathrm{SC}} \triangleq \sup _{\mathcal{U}, Q_{U X}} I_{\mathrm{SC}}\left(Q_{U X}\right)$. We also note the following dual expressions for

\footnotetext{
${ }^{1}$ The condition in [6] has a slightly different form to that in [6], which contains the additional constraint $I_{\widetilde{P}}(U ; X) \leq R_{0}$ and replaces the $[\cdot]^{+}$function in the objective by its argument. Both forms are given in [7], and their equivalence is proved therein. A simple way of seeing this equivalence is by noting that both expressions can be written as $0 \leq \min _{\widetilde{P}_{U X Y}} \max \left\{I_{\widetilde{P}}(U, X ; Y)-\left(R_{0}+R_{1}\right), I_{\widetilde{P}}(U ; X)-R_{0}\right\}$.
} 
(5)-(6) [6], [8]:

$$
\begin{gathered}
R_{1} \leq \sup _{s \geq 0, a(\cdot, \cdot)} \sum_{u, x, y} Q_{U X}(u, x) W(y \mid x) \log \frac{q(x, y)^{s} e^{a(u, x)}}{\sum_{\bar{x}} Q_{X \mid U}(\bar{x} \mid u) q(\bar{x}, y)^{s} e^{a(u, \bar{x})}} \\
R_{0} \leq \sup _{\rho_{1} \in[0,1], s \geq 0, a(\cdot, \cdot)} \sum_{u, x, y} Q_{U X}(u, x) W(y \mid x) \log \frac{\left(q(x, y)^{s} e^{a(u, x)}\right)^{\rho_{1}}}{\sum_{\bar{u}} Q_{U}(\bar{u})\left(\sum_{\bar{x}} Q_{X \mid U}(\bar{x} \mid \bar{u}) q(\bar{x}, y)^{s} e^{a(\bar{u}, \bar{x})}\right)^{\rho_{1}}}-\rho_{1} R_{1} .
\end{gathered}
$$

We outline the derivations of both the primal and dual expressions in Appendix $\mathrm{A}$

We note that $C_{\mathrm{SC}}$ is at least as high as Lapidoth's parallel coding rate [6]-[8], though it is not known whether it can be strictly higher. In [6], a refined version of superposition coding was shown to yield a rate improving on $I_{\mathrm{SC}}\left(Q_{U X}\right)$ for fixed $\left(\mathcal{U}, Q_{U X}\right)$, but the standard version will suffice for our purposes.

The above-mentioned technique of passing to the $k$-th order product alphabet is equally valid for the superposition coding achievable rate, and we denote the resulting achievable rate by $C_{\mathrm{SC}}^{(k)}$. The rate $C_{\mathrm{SC}}^{(2)}$ will be particularly important in this paper, and we will also use the analogous quantity $I_{\mathrm{SC}}^{(2)}\left(Q_{U X}\right)$ with a fixed input distribution $Q_{U X}$. Since the input alphabet of the product channel is $\mathcal{X}^{2}$, one might more precisely write the input distribution as $Q_{U X^{(2)}}$, but we omit this additional superscript. The choice $\mathcal{U}=\{0,1\}$ for the auxiliary alphabet will prove to be sufficient for our purposes.

\section{Converse}

Very few converse results have been provided for the mismatched decoding problem. Csiszár and Narayan [3] showed that $\lim _{k \rightarrow \infty} C_{\mathrm{LM}}^{(k)}=C_{\mathrm{M}}$ for erasures-only metrics, i.e. metrics such that $q(x, y)=\max _{x, y} q(x, y)$ for all $(x, y)$ such that $W(y \mid x)>0$. More recently, multi-letter converse results were given by Somekh-Baruch [11], yielding a general formula for the mismatched capacity in the sense of Verdú-Han [12]. However, these expressions are not computable.

The only general single-letter converse result presented in the literature is that of Balakirsky [13], who reported that $C_{\mathrm{LM}}=C_{\mathrm{M}}$ for binary-input DMCs. In the following section, we provide a counter-example showing that in fact the strict inequality $C_{\mathrm{M}}>C_{\mathrm{LM}}$ can hold even in this case.

\section{THE COUNTER-EXAMPLE}

The main claim of this paper is the following; the details are given in Section [II]

Counter-Example 1. Let $\mathcal{X}=\{0,1\}$ and $\mathcal{Y}=\{0,1,2\}$, and consider the channel and metric described by the entries of the $|\mathcal{X}| \times|\mathcal{Y}|$ matrices

$$
\boldsymbol{W}=\left[\begin{array}{ccc}
0.97 & 0.03 & 0 \\
0.1 & 0.1 & 0.8
\end{array}\right], \quad \boldsymbol{q}=\left[\begin{array}{ccc}
1 & 1 & 1 \\
1 & 0.5 & 1.36
\end{array}\right]
$$

Then the LM rate satisfies

$$
0.136874 \leq C_{\mathrm{LM}} \leq 0.136900 \text { nats/use }
$$




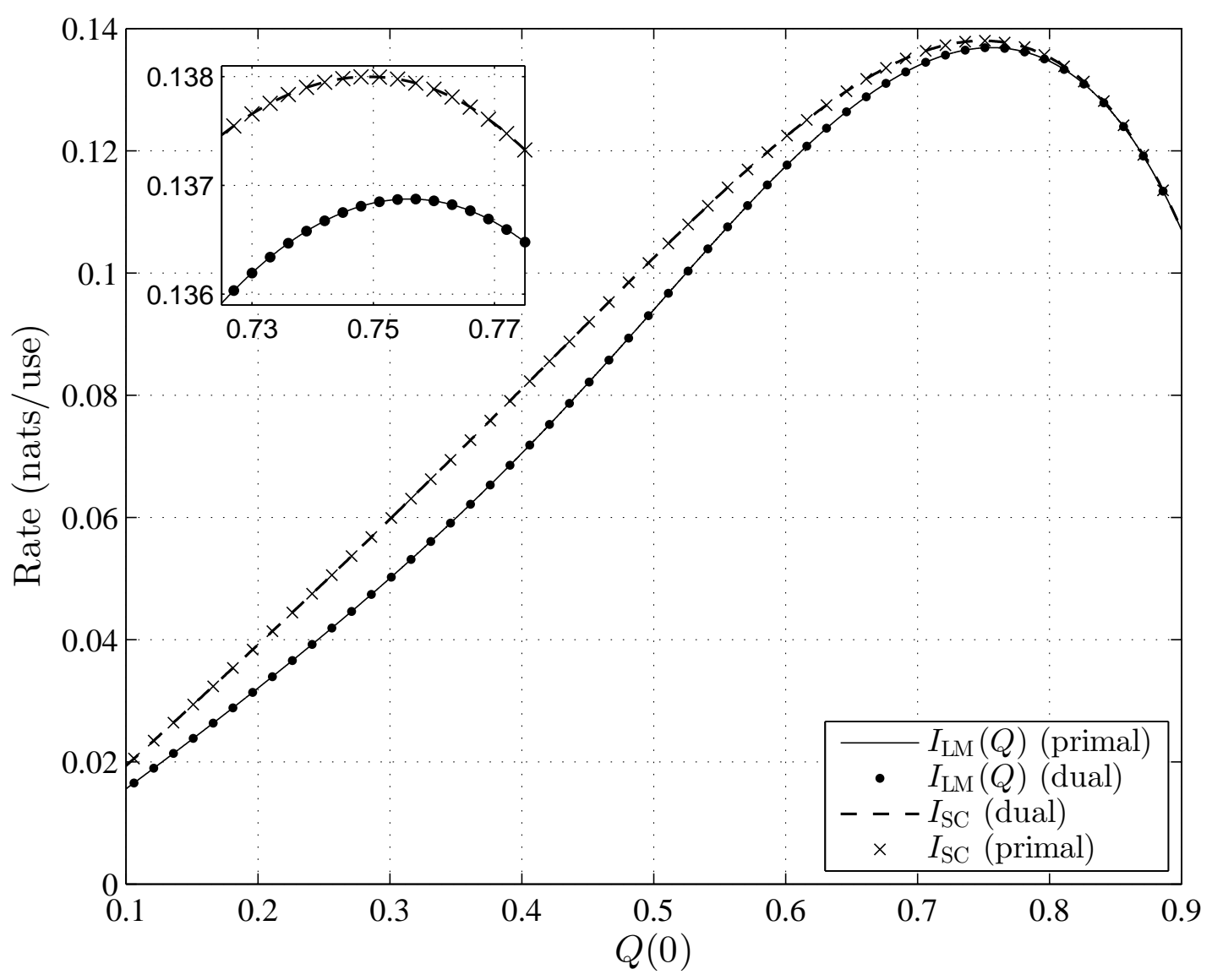

Figure 1: Numerical evaluations of the LM rate $I_{\mathrm{LM}}(Q)$ as a function of the (first entry of the) input distribution, and the corresponding superposition coding rate $I_{\mathrm{SC}}^{(2)}\left(Q_{U X}\right)$ using the construction described in Section $\amalg I I-D$, The matched capacity is $C \approx 0.4944$ nats/use, and is achieved by $Q(0) \approx 0.5398$.

whereas the superposition coding rate obtained by considering the second-order product of the channel is lower bounded by

$$
C_{\mathrm{SC}}^{(2)} \geq 0.137998 \text { nats/use }
$$

Consequently, we have $C_{\mathrm{M}}>C_{\mathrm{LM}}$.

We proceed by presenting various points of discussion.

Numerical Evaluations: While (10) and (11) are obtained using numerical computations, and the difference between the two is small, we will take care in ensuring that the gap is genuine, rather than being a matter of numerical accuracy. All of the code used in our computations is available online [14].

Figure 1 plots our numerical evaluations of $I_{\mathrm{LM}}(Q)$ and $I_{\mathrm{SC}}^{(2)}\left(Q_{U X}\right)$ for a range of input distributions; for the latter, $Q_{U X}$ is determined from $Q$ in a manner to be described in Section [II-D Note that this plot is only meant to help the reader visualize the results; it is not sufficient to establish Counter-Example 1 in itself. Nevertheless, it 
is reassuring to see that the curves corresponding to the primal and dual expressions are indistinguishable.

Our computations suggest that

$$
C_{\mathrm{LM}} \approx 0.136875 \text { nats/use, }
$$

and that the optimal input distribution is approximately

$$
Q=\left[\begin{array}{ll}
0.75597 & 0.24403
\end{array}\right] .
$$

The matched capacity is significantly higher than $C_{\mathrm{LM}}$, namely $C \approx 0.4944$ nats/use, with a corresponding input distribution approximately equal to [0.5398 0.4602]. As seen in the proof, the fact that the right-hand side of (10) exceeds that of (12) by $2.5 \times 10^{-5}$ is due to the use of (possibly crude) bounds on the loss in the rate when $Q$ is slightly suboptimal.

Other Achievable Rates: One may question whether (11) can be improved by considering $C_{\mathrm{SC}}^{(k)}$ for $k>2$. However, we were unable to find any such improvement when we tried $k=3$; see Section III-D for further discussion on this attempt. Similarly, we observed no improvement on (12) when we computed $I_{\mathrm{LM}}^{(2)}\left(Q^{(2)}\right)$ with a brute force search over $Q^{(2)} \in \mathcal{P}\left(\mathcal{X}^{2}\right)$ to two decimal places. Of course, it may still be that $C_{\mathrm{LM}}^{(k)}>C_{\mathrm{LM}}$ for some $k>2$, but optimizing $Q^{(k)}$ quickly becomes computationally difficult; even for $k=3$, the search space is 7-dimensional with no apparent convexity structure.

Our numerical findings also showed no improvement of the superposition coding rate $C_{\mathrm{SC}}$ for the original channel (as opposed to the product channel) over the $\mathrm{LM}$ rate $C_{\mathrm{LM}}$.

We were also able to obtain the achievable rate in (10) using Lapidoth's expurgated parallel coding rate [9] (or more precisely, its dual formulation from [6]) to the second-order product channel. In fact, this was done by taking the input distribution $Q_{U X}$ and the dual parameters $\left(s, a, \rho_{1}\right)$ used in (7)-(8) (see Section [II-D), and "transforming" them into parameters for the expurgated parallel coding ensemble that achieve an identical rate. Details are given in Appendix [C.

Choices of Channel and Metric: While the decoding metric in (9) may appear to be unusual, it should be noted that any decoding metric with $\max _{x, y} q(x, y)>0$ is equivalent to another metric yielding a matrix of this form with the first row and first column equal to one [5], [13].

One may question whether the LM rate can be improved for binary-input binary-output channels, as opposed to our ternary-output example. However, this is not possible, since for any such channel the LM rate is either equal to zero or the matched capacity, and in either case it coincides with the mismatched capacity [3].

Unfortunately, despite considerable effort, we have been unable to understand the analysis given in [13] in sufficient detail to identify any major errors therein. We also remark that for the vast majority of the examples we considered, $C_{\mathrm{LM}}$ was indeed greater than or equal to all other achievable rates that we computed. However, (9) was not the only counter-example, and others were found with $\min _{x, y} W(y \mid x)>0$ (in contrast with (9)). For example, a similar gap between the rates was observed when the first row of $\boldsymbol{W}$ in (9) was replaced by [0.97 0.020 .01 ] 


\section{Establishing COUNTER-EXAMPle 1}

While Counter-Example 1 is concerned with the specific channel and metric given in (9), we will present several results for more general channels with $\mathcal{X}=\{0,1\}$ and $\mathcal{Y}=\{0,1,2\}$ (and in some cases, arbitrary finite alphabets). To make some of the expressions more compact, we define $Q_{x} \triangleq Q(x), W_{x y} \triangleq W(y \mid x)$ and $q_{x y} \triangleq q(x, y)$ throughout this section.

\section{A. Auxiliary Lemmas}

The optimization of $I_{\mathrm{LM}}(Q)$ over $Q$ can be difficult, since $I_{\mathrm{LM}}(Q)$ is non-concave in $Q$ in general [10]. Since we are considering the case $|\mathcal{X}|=2$, this optimization is one-dimensional, and we thus resort to a straightforward brute-force search of $Q_{0}$ over a set of regularly-spaced points in [0,1]. To establish the upper bound in (10), we must bound the difference $C_{\mathrm{LM}}-I_{\mathrm{LM}}\left(Q_{0}\right)$ for the choice of $Q_{0}$ maximizing the LM rate among all such points. Lemma 2 below is used for precisely this purpose; before stating it, we present a preliminary result on the continuity of the binary entropy function $H_{2}(\alpha) \triangleq-\alpha \log \alpha-(1-\alpha) \log (1-\alpha)$.

It is well-known that for two distributions $Q$ and $Q^{\prime}$ on a common finite alphabet, we have $\left|H\left(Q^{\prime}\right)-H(Q)\right| \leq$ $\delta \log \frac{|\mathcal{X}|}{\delta}$ whenever $\left\|Q^{\prime}-Q\right\|_{1} \leq \delta[15$, Lemma 2.7]. The following lemma gives a refinement of this statement for the case that $|\mathcal{X}|=2$ and $\min \left\{Q_{0}^{\prime}, Q_{1}^{\prime}\right\}$ is no smaller than a predetermined constant.

Lemma 1. Let $Q^{\prime} \in \mathcal{P}(\mathcal{X})$ be a PMF on $\mathcal{X}=\{0,1\}$ such that $\min \left\{Q_{0}^{\prime}, Q_{1}^{\prime}\right\} \geq Q_{\min }^{\prime}$ for some $Q_{\min }^{\prime}>0$. For any $P M F Q \in \mathcal{P}(\mathcal{X})$ such that $\left|Q_{0}-Q_{0}^{\prime}\right| \leq \delta$ (or equivalently, $\left|Q_{1}-Q_{1}^{\prime}\right| \leq \delta$ ), we have

$$
\left|H\left(Q^{\prime}\right)-H(Q)\right| \leq \delta \log \frac{1-Q_{\min }^{\prime}}{Q_{\min }^{\prime}} .
$$

Proof: Set $\Delta \triangleq Q_{0}-Q_{0}^{\prime}$. Since $H_{2}(\cdot)$ is concave, the straight line tangent to a given point always lies above the function itself. Assuming without loss of generality that $Q_{0}^{\prime} \leq 0.5$, we have

$$
\begin{aligned}
\left|H_{2}\left(Q_{0}^{\prime}+\Delta\right)-H_{2}\left(Q_{0}^{\prime}\right)\right| & \leq\left.|\Delta| \cdot \frac{d H_{2}}{d \alpha}\right|_{\alpha=Q_{0}^{\prime}} \\
& =|\Delta| \log \frac{1-Q_{0}^{\prime}}{Q_{0}^{\prime}} .
\end{aligned}
$$

The desired result follows since $\frac{1-Q_{0}^{\prime}}{Q_{0}^{\prime}}$ is decreasing in $Q_{0}^{\prime}$, and since $Q_{0}^{\prime} \geq Q_{\min }^{\prime}$ and $|\Delta| \leq \delta$ by assumption.

The following lemma builds on the preceding lemma, and is key to establishing Counter-Example 1.

Lemma 2. For any binary-input mismatched DMC, we have the following under the setup of Lemma 1 .

$$
I_{\mathrm{LM}}(Q) \geq I_{\mathrm{LM}}\left(Q^{\prime}\right)-\delta \log \frac{1-Q_{\min }^{\prime}}{Q_{\min }^{\prime}}-\frac{\delta \log 2}{Q_{\min }^{\prime}} .
$$

Proof: The bound in (17) is trivial when $I_{\mathrm{LM}}\left(Q^{\prime}\right)=0$, so we consider the case $I_{\mathrm{LM}}\left(Q^{\prime}\right)>0$. Observing that $Q(x)>0$ for $x \in\{0,1\}$, we can make the change of variable $a(x)=\log \frac{e^{\tilde{a}(x)}}{Q(x)}$ (i.e. $\left.e^{\tilde{a}(x)}=Q(x) e^{a(x)}\right)$ in $(4)$ to obtain

$$
I_{\mathrm{LM}}(Q)=\sup _{s \geq 0, \tilde{a}(\cdot)} \sum_{x, y} Q(x) W(y \mid x) \log \frac{q(x, y)^{s} e^{\tilde{a}(x)}}{Q(x) \sum_{\bar{x}} q(\bar{x}, y)^{s} e^{\tilde{a}(\bar{x})}}
$$


which can equivalently be written as

$$
I_{\mathrm{LM}}\left(Q^{\prime}\right)=H\left(Q^{\prime}\right)-\inf _{s \geq 0, \tilde{a}(\cdot)} \sum_{x, y} Q^{\prime}(x) W(y \mid x) \log \left(1+\frac{q(\bar{x}, y)^{s} e^{\tilde{a}(\bar{x})}}{q(x, y)^{s} e^{\tilde{a}(x)}}\right),
$$

where $\bar{x} \in\{0,1\}$ denotes the unique symbol differing from $x \in\{0,1\}$.

The following arguments can be simplified when the infimum is achieved, but for completeness we consider the general case. Let $\left(s_{k}, \tilde{a}_{k}\right)$ be a sequence of parameters such that

$$
H\left(Q^{\prime}\right)-\lim _{k \rightarrow \infty} \sum_{x, y} Q^{\prime}(x) W(y \mid x) \log \left(1+\frac{q(\bar{x}, y)^{s_{k}} e^{\tilde{a}_{k}(\bar{x})}}{q(x, y)^{s_{k}} e^{\tilde{a}_{k}(x)}}\right)=I_{\mathrm{LM}}\left(Q^{\prime}\right) .
$$

Since the argument to the logarithm in (20) is no smaller than one, and since $H\left(Q^{\prime}\right) \leq \log 2$ by the assumption that the input alphabet is binary, we have for $x=0,1$ and sufficiently large $k$ that

$$
\sum_{y} Q^{\prime}(x) W(y \mid x) \log \left(1+\frac{q(\bar{x}, y)^{s_{k}} e^{\tilde{a}_{k}(\bar{x})}}{q(x, y)^{s_{k}} e^{\tilde{a}_{k}(x)}}\right) \leq \log 2,
$$

since otherwise the left-hand side of 20) would be non-positive, in contradiction with the fact that we are considering the case $I_{\mathrm{LM}}\left(Q^{\prime}\right)>0$. Using the assumption $\min \left\{Q_{0}^{\prime}, Q_{1}^{\prime}\right\} \geq Q_{\min }^{\prime}$, we can weaken (21) to

$$
\sum_{y} W(y \mid x) \log \left(1+\frac{q(\bar{x}, y)^{s_{k}} e^{\tilde{a}_{k}(\bar{x})}}{q(x, y)^{s_{k}} e^{\tilde{a}_{k}(x)}}\right) \leq \frac{\log 2}{Q_{\min }^{\prime}} .
$$

We now have the following:

$$
\begin{aligned}
I_{\mathrm{LM}}(Q) & \geq H(Q)-\limsup _{k \rightarrow \infty} \sum_{x, y} Q(x) W(y \mid x) \log \left(1+\frac{q(\bar{x}, y)^{s_{k}} e^{\tilde{a}_{k}(\bar{x})}}{q(x, y)^{s_{k}} e^{\tilde{a}_{k}(x)}}\right) \\
& \geq H\left(Q^{\prime}\right)-\limsup _{k \rightarrow \infty} \sum_{x} Q(x) \sum_{y} W(y \mid x) \log \left(1+\frac{q(\bar{x}, y)^{s_{k}} e^{\tilde{a}_{k}(\bar{x})}}{q(x, y)^{s_{k}} e^{\tilde{a}_{k}(x)}}\right)-\delta \log \frac{1-Q_{\min }^{\prime}}{Q_{\min }^{\prime}} \quad(24) \\
& =H\left(Q^{\prime}\right)-\limsup _{k \rightarrow \infty} \sum_{x}\left(Q(x)+Q^{\prime}(x)-Q^{\prime}(x)\right) \sum_{y} W(y \mid x) \log \left(1+\frac{q(\bar{x}, y)^{s_{k}} e^{\tilde{a}_{k}(\bar{x})}}{q(x, y)^{s_{k}} e^{\tilde{a}_{k}(x)}}\right)-\delta \log \frac{1-Q_{\min }^{\prime}}{Q_{\min }^{\prime}} \\
& \geq H\left(Q^{\prime}\right)-\limsup _{k \rightarrow \infty} \sum_{x} Q^{\prime}(x) \sum_{y} W(y \mid x) \log \left(1+\frac{q(\bar{x}, y)^{s_{k}} e^{\tilde{a}_{k}(\bar{x})}}{q(x, y)^{s_{k}} e^{\tilde{a}_{k}(x)}}\right)-\delta \log \frac{1-Q_{\min }^{\prime}}{Q_{\min }^{\prime}}-\frac{\delta \log 2}{Q_{\min }^{\prime}} \\
& =I_{\mathrm{LM}}\left(Q^{\prime}\right)-\delta \log \frac{1-Q_{\min }^{\prime}}{Q_{\min }^{\prime}}-\frac{\delta \log 2}{Q_{\min }^{\prime}},
\end{aligned}
$$

where (23) follows by replacing the infimum in (19) by the particular sequence of parameters $\left(s_{k}, \tilde{a}_{k}\right)$ and taking the limsup, (24) follows from Lemma 1, (26) follows by applying (22) for the $x$ value where $Q(x) \geq Q^{\prime}(x)$ and lower bounding the logarithm by zero for the other $x$ value, and (27) follows from (20).

\section{B. Establishing the Upper Bound in (10)}

As mentioned in the previous subsection, we optimize $Q$ by performing a brute force search over a set of regularly spaced points, and then using Lemma 2 to bound the difference $C_{\mathrm{LM}}-I_{\mathrm{LM}}(Q)$. We let the input distribution therein be $Q^{\prime}=\arg \max _{Q} I_{\mathrm{LM}}(Q)$. Note that this maximum is always achieved, since $I_{\mathrm{LM}}$ is continuous and bounded [3]. If there are multiple maximizers, we choose one arbitrarily among them. 
To apply Lemma 2, we need a constant $Q_{\min }^{\prime}$ such that $\min \left\{Q_{0}^{\prime}, Q_{1}^{\prime}\right\} \geq Q_{\min }^{\prime}$. We present a straightforward choice based on the lower bound on the left-hand side of (10) (proved in Section $\left[11-\mathrm{C}\right.$ ). By choosing $Q_{\min }^{\prime}$ such that even the mutual information $I(X ; Y)$ is upper bounded by the left-hand side of (10) when $\min \left\{Q_{0}^{\prime}, Q_{1}^{\prime}\right\}<Q_{\min }^{\prime}$, we see from the simple identity $I_{\mathrm{LM}}(Q) \leq I(X ; Y)[3]$ that $Q$ cannot maximize $I_{\mathrm{LM}}$. For the example under consideration (see (9)), the choice $Q_{\min }^{\prime}=0.042$ turns out to be sufficient, and in fact yields $I(X ; Y) \leq 0.135$. This can be verified by computing $I(X ; Y)$ to be (approximately) $0.0917,0.4919$ and 0.1348 for $Q_{0}=0.042, Q_{0}=0.5$ and $Q_{0}=1-0.042$ respectively, and then using the concavity of $I(X ; Y)$ in $Q$ to handle $Q_{0} \in[0,0.042) \cup(1-0.042,1]$.

Let $h \triangleq 10^{-5}$, and suppose that we evaluate $I_{\mathrm{LM}}(Q)$ for each $Q_{0}$ in the set

$$
\mathcal{A} \triangleq\left\{Q_{\min }^{\prime}, Q_{\min }^{\prime}+h, \ldots, 1-Q_{\min }^{\prime}-h, 1-Q_{\min }^{\prime}\right\} .
$$

Since the optimal input distribution $Q^{\prime}$ corresponds to some $Q_{0}^{\prime} \in\left[Q_{\min }^{\prime}, 1-Q_{\min }^{\prime}\right]$, we conclude that there exists some $Q_{0} \in \mathcal{A}$ such that $\left|Q_{0}^{\prime}-Q_{0}\right| \leq \frac{h}{2}$. Substituting $\delta=\frac{h}{2}=0.5 \times 10^{-5}$ and $Q_{\min }^{\prime}=0.042$ into (17), we conclude that

$$
\max _{Q_{0} \in \mathcal{A}} I_{\mathrm{LM}}(Q) \geq C_{\mathrm{LM}}-0.982 \times 10^{-4} .
$$

We now describe our techniques for evaluating $I_{\mathrm{LM}}(Q)$ for a fixed choice of $Q$. This is straightforward in principle, since the corresponding optimization problem is convex whether we use the primal expression in (3) or the dual expression in (4). Nevertheless, since we need to test a large number of $Q_{0}$ values, we make an effort to find a reasonably efficient method.

We avoid using the dual expression in (4), since it is a maximization problem; thus, if the final optimization parameters obtained differ slightly from the true optimal parameters, they will only provide a lower bound on $I_{\mathrm{LM}}(Q)$. In contrast, the result that we seek is an upper bound. We also avoid evaluating (3) directly, since the equality constraints in the optimization problem could, in principle, be sensitive to numerical precision errors.

Of course, there are many ways to circumvent these problems and provide rigorous bounds on the suboptimality of optimization procedures, including a number of generic solvers. We instead take a different approach, and reduce the primal optimization in (10) to a scalar minimization problem by eliminating the constraints one-by-one. This minimization will contain no equality constraints, and thus minor variations in the optimal parameter will still produce a valid upper bound.

We first note that the inequality constraint can be replaced by an equality whenever $I_{\mathrm{LM}}(Q)>0$ [3, Lemma 1], which is certainly the case for the present example. Moreover, since the $X$-marginal is constrained to equal $Q$, we can let the minimization be over $\mathcal{P}(\mathcal{Y} \mid \mathcal{X})$ instead of $\mathcal{P}(\mathcal{X} \times \mathcal{Y})$, yielding

$$
I_{\mathrm{LM}}(Q)=\min _{\substack{\widetilde{W} \in \mathcal{P}(\mathcal{Y} \mid \mathcal{X}): \widetilde{P}_{Y}=P_{Y} \\ \mathbb{E}_{Q \times \widetilde{W}}[\log q(X, Y)]=\mathbb{E}_{P}[\log q(X, Y)]}} I_{Q \times \widetilde{W}}(X ; Y),
$$

where $\widetilde{P}_{Y}(y) \triangleq \sum_{x} Q(x) \widetilde{W}(y \mid x)$ (recall also that $P_{X Y}=Q \times W$ ). Let us fix a conditional distribution $\widetilde{W}$ satisfying 
the specified constraints, and write $\widetilde{W}_{x y} \triangleq \widetilde{W}(y \mid x)$. The analogous matrix to $\boldsymbol{W}$ in (9) can be written as follows:

$$
\widetilde{\boldsymbol{W}}=\left[\begin{array}{ccc}
\widetilde{W}_{00} & \widetilde{W}_{01} & 1-\widetilde{W}_{00}-\widetilde{W}_{01} \\
\widetilde{W}_{10} & \widetilde{W}_{11} & 1-\widetilde{W}_{10}-\widetilde{W}_{11}
\end{array}\right] .
$$

Since $\widetilde{P}_{Y}=P_{Y}$ implies $H\left(\widetilde{P}_{Y}\right)=H\left(P_{Y}\right)$, we can write the objective in (30) as

$$
\begin{aligned}
I_{Q \times \widetilde{W}}(X ; Y)=H\left(P_{Y}\right) & -H_{Q \times \widetilde{W}}(Y \mid X) \\
=H\left(P_{Y}\right) & +Q_{0}\left(\widetilde{W}_{00} \log \widetilde{W}_{00}+\widetilde{W}_{01} \log \widetilde{W}_{01}+\left(1-\widetilde{W}_{00}-\widetilde{W}_{01}\right) \log \left(1-\widetilde{W}_{00}-\widetilde{W}_{01}\right)\right) \\
& +Q_{1}\left(\widetilde{W}_{10} \log \widetilde{W}_{10}+\widetilde{W}_{11} \log \widetilde{W}_{11}+\left(1-\widetilde{W}_{10}-\widetilde{W}_{11}\right) \log \left(1-\widetilde{W}_{10}-\widetilde{W}_{11}\right)\right)
\end{aligned}
$$

We now show that the equality constraints can be used to express each $\widetilde{W}_{x y}$ in terms of $\widetilde{W}_{10}$. Using $\widetilde{P}_{Y}(y)=P_{Y}(y)$ for $y=0,1$, along with the constraint containing the decoding metric, we have

$$
\begin{gathered}
Q_{0} \widetilde{W}_{00}+Q_{1} \widetilde{W}_{10}=P_{Y}(0) \\
Q_{0} \widetilde{W}_{01}+Q_{1} \widetilde{W}_{11}=P_{Y}(1) \\
Q_{1}\left(\widetilde{W}_{11} \log q_{11}+\left(1-\widetilde{W}_{10}-\widetilde{W}_{11}\right) \log q_{12}\right)=\mathbb{E}_{P}[\log q(X, Y)],
\end{gathered}
$$

where in (36) we used the fact that $\log q(x, y)=0$ for four of the six $(x, y)$ pairs (see (96). Re-arranging (34)-(36), we obtain

$$
\begin{aligned}
\widetilde{W}_{00} & =\frac{P_{Y}(0)-Q_{1} \widetilde{W}_{10}}{Q_{0}} \\
\widetilde{W}_{01} & =\frac{P_{Y}(1)-Q_{1} \widetilde{W}_{11}}{Q_{0}} \\
\widetilde{W}_{11} & =\frac{1}{\log q_{11}-\log q_{12}}\left(\frac{\mathbb{E}_{P}[\log q(X, Y)]}{Q_{1}}-\left(1-\widetilde{W}_{10}\right) \log q_{12}\right),
\end{aligned}
$$

and substituting (39) into 38 yields

$$
\widetilde{W}_{01}=\frac{1}{Q_{0}}\left(P_{Y}(1)-\frac{1}{\log q_{11}-\log q_{12}}\left(\mathbb{E}_{P}[\log q(X, Y)]-Q_{1}\left(1-\widetilde{W}_{10}\right) \log q_{12}\right)\right) .
$$

We have thus written each entry of $(33)$ in terms of $\widetilde{W}_{10}$, and we are left with a one-dimensional optimization problem. However, we must still ensure that the constraints $\widetilde{W}_{x y} \in[0,1]$ are satisfied for all $(x, y)$. Since each $\widetilde{W}_{x y}$ is an affine function of $\widetilde{W}_{10}$, these constraints are each of the form $\underline{W}^{(x, y)} \leq \widetilde{W}_{10} \leq \bar{W}^{(x, y)}$, and the overall optimization is given by

$$
\min _{\underline{W} \leq \widetilde{W}_{10} \leq \bar{W}} f\left(\widetilde{W}_{10}\right)
$$

where $f(\cdot)$ denotes the right-hand side of (33) upon substituting (37), (39) and (40), and the lower and upper limits are given by $\underline{W} \triangleq \max _{x, y} \underline{W}^{(x, y)}$ and $\bar{W} \triangleq \min _{x, y} \bar{W}^{(x, y)}$. Note that the minimization region is non-empty, since $\widetilde{\boldsymbol{W}}=\boldsymbol{W}$ is always feasible. In principle one could observe $\underline{W}=\bar{W}=W_{10}$, but in the present example we found that $\underline{W}<\bar{W}$ for every choice of $Q_{0}$ that we used.

The optimization problem in (41) does not appear to permit an explicit solution. However, we can efficiently compute the solution to high accuracy using standard one-dimensional optimization methods. Since the convexity 
of any optimization problem is preserved by the elimination of equality constraints [16, Sec. 4.2.4], and since the optimization problem in (30) is convex for any given $Q$, we conclude that $f(\cdot)$ is a convex function. Its derivative is easily computed by noting that

$$
\frac{d}{d z}(\alpha z+\beta) \log (\alpha z+\beta)=\alpha+\alpha \log (\alpha z+\beta)
$$

for all $\alpha, \beta$ and $z$ yielding a positive argument to the logarithm. We can thus perform a bisection search as follows, where $f^{\prime}(\cdot)$ denotes the derivative of $f$, and $\epsilon$ is a termination parameter:

1) Set $i=0, \underline{W}^{(0)}=\underline{W}$ and $\bar{W}^{(0)}=\bar{W}$;

2) Set $W_{\text {mid }}=\frac{1}{2}\left(\underline{W}^{(i)}+\bar{W}^{(i)}\right)$; if $f^{\prime}\left(W_{\text {mid }}\right) \geq 0$ then set $\underline{W}^{(i+1)}=\underline{W}^{(i)}$ and $\bar{W}^{(i+1)}=W_{\text {mid }}$; otherwise set $\underline{W}^{(i+1)}=W_{\text {mid }}$ and $\bar{W}^{(i+1)}=\bar{W}^{(i)}$;

3) If $\left|f^{\prime}\left(W_{\text {mid }}\right)\right| \leq \epsilon$ then terminate; otherwise increment $i$ and return to Step 2.

As mentioned previously, we do not need to find the exact solution to $\underline{41}$, since any value of $\widetilde{W}_{10} \in[\underline{W}, \bar{W}]$ yields a valid upper bound on $I_{\mathrm{LM}}(Q)$. However, we must choose $\epsilon$ sufficiently small so that the bound in (10) is established. We found $\epsilon=10^{-6}$ to suffice.

We implemented the preceding techniques in $\mathrm{C}$ (see [14] for the code) to upper bound $I_{\mathrm{LM}}(Q)$ for each $Q_{0} \in \mathcal{A}$; see Figure 11 As stated following Counter-Example 1, we found the highest value of $I_{\mathrm{LM}}(Q)$ to be the right-hand side of (12), corresponding to the input distribution in (13). We found the corresponding minimizing parameter in (41) to be roughly $\widetilde{W}_{10}=0.4252347$.

Instead of directly adding $10^{-4}$ to (12) in accordance with (29), we obtain a refined estimate by "updating" our estimate of $Q_{\min }^{\prime}$. Specifically, using (29) and observing the values in Figure 1, we can conclude that the optimal value of $Q_{0}$ lies in the range $[0.7,0.8]$ (we are being highly conservative here). Thus, setting $Q_{\min }^{\prime}=0.2$ and using the previously chosen value $\delta=0.5 \times 10^{-5}$, we obtain the following refinement of [29]:

$$
\max _{Q_{0} \in \mathcal{A}} I_{\mathrm{LM}}(Q) \geq C_{\mathrm{LM}}-2.43 \times 10^{-5} .
$$

Since our implementation in $\mathrm{C}$ is based on floating-point calculations, the final values may have precision errors. We therefore checked our numbers using Mathematica's arbitrary-precision arithmetic framework [17], which allows one to work with exact expressions that can then be displayed to arbitrarily many decimal places. More precisely, we loaded the values of $\widetilde{W}_{10}$ into Mathematica and rounded them to 12 decimal places (this is allowed, since any value of $\widetilde{W}_{10}$ yields a valid upper bound). Using the exact values of all other quantities (e.g. $Q$ and $W$ ), we performed an evaluation of $f\left(\widetilde{W}_{10}\right)$ in (41), and compared it to the corresponding value of $I_{\mathrm{LM}}(Q)$ produced by the $\mathrm{C}$ program. The maximum discrepancy across all of the values of $Q_{0}$ was less than $2.1 \times 10^{-12}$. Our final bound in (10) was obtained by adding $2.5 \times 10^{-5}$ (which is, of course, higher than $2.43 \times 10^{-5}+2.1 \times 10^{-12}$ ) to the right-hand side of (12). 


\section{Establishing the Lower Bound in (10)}

For the lower bound, we can afford to be less careful than we were in establishing the upper bound; all we need is a suitable choice of $Q$ and the parameters $(s, a)$ in (4). We choose $Q$ as in (13), along with the following:

$$
\begin{aligned}
s & =9.031844 \\
\boldsymbol{a} & =\left[\begin{array}{ll}
0.355033 & -0.355033
\end{array}\right],
\end{aligned}
$$

In Appendix B we provide details on how these parameters were obtained, though the desired lower bound can readily be verified without knowing such details.

Using these values, we evaluated the objective in (4) using Mathematica's arbitrary-precision arithmetic framework [17], thus eliminating the possibility of arithmetic precision errors. See [14] for the relevant C and Mathematica code.

\section{Establishing the Lower Bound in (11)}

We establish the lower bound in (11) by setting $\mathcal{U}=\{0,1\}$ and forming a suitable choice of $Q_{U X}$, and then using the dual expressions in (7)-(8) to lower bound $I_{\mathrm{SC}}^{(2)}\left(Q_{U X}\right)$.

1) Choice of Input Distribution: Let $Q=\left[Q_{0} Q_{1}\right]$ be some input distribution on $\mathcal{X}$, and define the corresponding product distribution on $\mathcal{X}^{2}$ as

$$
Q^{(2)}=\left[\begin{array}{llll}
Q_{0}^{2} & Q_{0} Q_{1} & Q_{0} Q_{1} & Q_{1}^{2}
\end{array}\right],
$$

where the order of the inputs is $(0,0),(0,1),(1,0),(1,1)$. Consider now the following choice of superposition coding parameters for the second-order product channel $\left(W^{(2)}, q^{(2)}\right)$ :

$$
\begin{aligned}
& Q_{U}=\left[\begin{array}{ll}
1-Q_{1}^{2} & Q_{1}^{2}
\end{array}\right] \\
& Q_{X \mid U=0}=\frac{1}{1-Q_{1}^{2}}\left[\begin{array}{llll}
Q_{0}^{2} & Q_{0} Q_{1} & Q_{0} Q_{1} & 0
\end{array}\right] \\
& Q_{X \mid U=1}=\left[\begin{array}{llll}
0 & 0 & 0 & 1
\end{array}\right] \text {. }
\end{aligned}
$$

This choice yields an $X$-marginal $Q_{X}$ precisely given by (46), and it is motivated by the empirical observation from [6] that choices of $Q_{U X}$ where $Q_{X \mid U=1}$ and $Q_{X \mid U=2}$ have disjoint supports tend to provide good rates. We let the single-letter distribution $Q=\left[Q_{0} Q_{1}\right]$ be

$$
Q=\left[\begin{array}{ll}
0.749 & 0.251
\end{array}\right] \text {. }
$$

which we chose based on a simple brute force search (see Figure 1). Note that this choice is similar to that in (13), but not identical.

One may question whether the choice of the supports of $Q_{X \mid U=0}$ and $Q_{X \mid U=1}$ in (48)-(49) is optimal. For example, a similar construction might set $Q_{U}(0)=Q_{0}^{2}+Q_{0} Q_{1}$, and then replace (48)-(49) by normalized versions of $\left[\begin{array}{llll}Q_{0}^{2} & Q_{0} Q_{1} & 0 & 0\end{array}\right]$ and $\left[\begin{array}{lllll}0 & 0 & Q_{0} Q_{1} & Q_{1}^{2}\end{array}\right]$. However, after performing a brute force search over the possible support patterns (there are no more than $2^{4}$, and many can be ruled out by symmetry considerations), we found the above 
pattern to be the only one to give an improvement on $I_{\mathrm{LM}}$, at least for the choices of input distribution in (13) and (50). In fact, even after setting $|\mathcal{U}|=3$, considering the third-order product channel $\left(W^{(3)}, q^{(3)}\right)$, and performing a similar brute force search over the support patterns (of which there are no more than $3^{8}$ ), we were unable to obtain an improvement on (11).

2) Choices of Optimization Parameters: We now specify the choices of the dual parameters in (7)-(8). In Appendix B, we give details of how these parameters were obtained. We claim that the choice

$$
\left(R_{0}, R_{1}\right)=(0.0356005,0.2403966)
$$

is permitted; observe that summing these two values and dividing by two (since we are considering the product channel) yields (11). These values can be verified by setting the parameters as follows: On the right-hand side of (7), set

$$
\begin{aligned}
s & =9.4261226 \\
\boldsymbol{a} & =\left[\begin{array}{cccc}
0.4817048 & -0.2408524 & -0.2408524 & 0 \\
0 & 0 & 0 & 0
\end{array}\right],
\end{aligned}
$$

and on the right-hand side of $[8]$, set

$$
\begin{aligned}
\rho_{1} & =0.7587516 \\
s & =9.3419338 \\
\boldsymbol{a} & =\left[\begin{array}{cccc}
0.7186926 & -0.0488036 & -0.0488036 & 0 \\
0 & 0 & 0 & -0.6210855
\end{array}\right] .
\end{aligned}
$$

Once again, we evaluated (7)-(8) using Mathematica's arbitrary-precision arithmetic framework [17], thus ensuring the validity of [11). See [14] for the relevant $\mathrm{C}$ and Mathematica code.

\section{CONCLUSION AND Discussion}

We have used our numerical findings, along with an analysis of the gap to suboptimality for slightly suboptimal input distributions, to show that it is possible for $C_{\mathrm{M}}$ to exceed $C_{\mathrm{LM}}$ even for binary-input mismatched DMCs. This is in contrast with the claim in [13] that $C_{\mathrm{M}}=C_{\mathrm{LM}}$ for such channels.

An interesting direction for future research is to find a purely theoretical proof of Counter-Example 1, the nonconcavity of $I_{\mathrm{LM}}(Q)$ observed in Figure 1 may play a role in such an investigation. Furthermore, it would be of significant interest to develop a better understanding of [13], including which parts may be incorrect, under what conditions the converse remains valid, and in the remaining cases, whether a valid converse lying in between the LM rate and matched capacity can be inferred.

\section{APPENDIX A}

\section{DERIVATIONS OF THE SUPERPOSITION CODING RATES}

Here we outline how the superposition coding rates in (5)-(8) are obtained using the techniques of [6], [7]. The equivalence of the primal and dual formulations can also be proved using techniques presented in [6]. 


\section{A. Preliminary Definitions and Results}

The parameters of the random-coding ensemble are a finite auxiliary alphabet $\mathcal{U}$, an auxiliary codeword distribution $P_{\boldsymbol{U}}$, and a conditional codeword distribution $P_{\boldsymbol{X} \mid \boldsymbol{U}}$. An auxiliary codebook $\left\{\boldsymbol{U}^{(i)}\right\}_{i=1}^{M_{0}}$ with $M_{0}$ codewords is generated, with each auxiliary codeword independently distributed according to $P_{\boldsymbol{U}}$. For each $i=1, \ldots, M_{0}$, a codebook $\left\{\boldsymbol{X}^{(i, j)}\right\}_{j=1}^{M_{1}}$ with $M_{1}$ codewords is generated, with each codeword conditionally independently distributed according to $P_{\boldsymbol{X} \mid \boldsymbol{U}}\left(\cdot \mid \boldsymbol{U}^{(i)}\right)$. The message $m$ at the input to the encoder is indexed as $\left(m_{0}, m_{1}\right)$, and for any such pair, the corresponding transmitted codeword is $\boldsymbol{X}^{\left(m_{0}, m_{1}\right)}$. Thus, the overall number of messages is $M=M_{1} M_{2}$, yielding a rate of $R=R_{1}+R_{2}$. More compactly, we have

$$
\left\{\left(\boldsymbol{U}^{(i)},\left\{\boldsymbol{X}^{(i, j)}\right\}_{j=1}^{M_{1}}\right)\right\}_{i=1}^{M_{0}} \sim \prod_{i=1}^{M_{0}}\left(P_{\boldsymbol{U}}\left(\boldsymbol{u}^{(i)}\right) \prod_{j=1}^{M_{1}} P_{\boldsymbol{X} \mid \boldsymbol{U}}\left(\boldsymbol{x}^{(i, j)} \mid \boldsymbol{u}^{(i)}\right)\right) .
$$

We assume without loss of generality that message $(1,1)$ is transmitted, and we write $\boldsymbol{U}$ and $\boldsymbol{X}$ in place of $\boldsymbol{U}^{(1)}$ and $\boldsymbol{X}^{(1,1)}$ respectively. We write $\widetilde{\boldsymbol{X}}$ to denote an arbitrary codeword $\boldsymbol{X}^{(1, j)}$ with $j \neq 1$. Moreover, we let $\overline{\boldsymbol{U}}$ denote an arbitrary auxiliary codeword $\boldsymbol{U}^{(i)}$ with $i \neq 1$, let $\overline{\boldsymbol{X}}^{(j)}\left(j=1, \cdots, M_{1}\right)$ denote the corresponding codeword $\boldsymbol{X}^{(i, j)}$, and let $\overline{\boldsymbol{X}}$ denote $\overline{\boldsymbol{X}}^{(j)}$ for an arbitrary choice of $j$. Thus, defining $\boldsymbol{Y}$ to be the channel output, we have

$$
(\boldsymbol{U}, \boldsymbol{X}, \boldsymbol{Y}, \tilde{\boldsymbol{X}}, \overline{\boldsymbol{U}}, \overline{\boldsymbol{X}}) \sim P_{\boldsymbol{U}}(\boldsymbol{u}) P_{\boldsymbol{X} \mid \boldsymbol{U}}(\boldsymbol{x} \mid \boldsymbol{u}) W^{n}(\boldsymbol{y} \mid \boldsymbol{x}) P_{\boldsymbol{X} \mid \boldsymbol{U}}(\widetilde{\boldsymbol{x}} \mid \boldsymbol{u}) P_{\boldsymbol{U}}(\overline{\boldsymbol{u}}) P_{\boldsymbol{X} \mid \boldsymbol{U}}(\overline{\boldsymbol{x}} \mid \overline{\boldsymbol{u}}) .
$$

The decoder estimates $\hat{m}=\left(\hat{m}_{0}, \hat{m}_{1}\right)$ according to the decoding rule in (1). We define the following error events:

(Type 0) $\quad q^{n}\left(\boldsymbol{X}^{(i, j)}, \boldsymbol{Y}\right) \geq q^{n}(\boldsymbol{X}, \boldsymbol{Y})$ for some $i \neq 1, j$;

(Type 1) $\quad q^{n}\left(\boldsymbol{X}^{(1, j)}, \boldsymbol{Y}\right) \geq q^{n}(\boldsymbol{X}, \boldsymbol{Y})$ for some $j \neq 1$.

The probabilities of these events are denoted by $\bar{p}_{\mathrm{e}, 0}\left(n, M_{0}, M_{1}\right)$ and $\bar{p}_{\mathrm{e}, 1}\left(n, M_{1}\right)$ respectively. The overall randomcoding error probability $\bar{p}_{\mathrm{e}}\left(n, M_{0}, M_{1}\right)$ clearly satisfies $\bar{p}_{\mathrm{e}} \leq \bar{p}_{\mathrm{e}, 0}+\bar{p}_{\mathrm{e}, 1}$.

We begin by deriving the following non-asymptotic bounds:

$$
\begin{gathered}
\bar{p}_{\mathrm{e}, 0}\left(n, M_{0}, M_{1}\right) \leq \mathbb{E}\left[\min \left\{1,\left(M_{0}-1\right) \mathbb{E}\left[\min \left\{1, M_{1} \mathbb{P}\left[\frac{q^{n}(\overline{\boldsymbol{X}}, \boldsymbol{Y})}{q^{n}(\boldsymbol{X}, \boldsymbol{Y})} \geq 1 \mid \overline{\boldsymbol{U}}\right]\right\} \mid \boldsymbol{U}, \boldsymbol{X}, \boldsymbol{Y}\right]\right\}\right] \\
\bar{p}_{\mathrm{e}, 1}\left(n, M_{1}\right) \leq \mathbb{E}\left[\min \left\{1,\left(M_{1}-1\right) \mathbb{P}\left[\frac{q^{n}(\widetilde{\boldsymbol{X}}, \boldsymbol{Y})}{q^{n}(\boldsymbol{X}, \boldsymbol{Y})} \geq 1 \mid \boldsymbol{U}, \boldsymbol{X}, \boldsymbol{Y}\right]\right\}\right] .
\end{gathered}
$$

We will see that (59) recovers the rate conditions in (6) and (8), whereas (60) recovers those in (5) and (7). We focus on the type- 0 event throughout the appendix, since the type- 1 event is simpler, and is handled using standard techniques associated with the case of independent codewords.

To derive (59), we first note that

$$
\bar{p}_{\mathrm{e}, 0}=\mathbb{P}\left[\bigcup_{i \neq 1, j \neq 1}\left\{\frac{q^{n}\left(\boldsymbol{X}^{(i, j)}, \boldsymbol{Y}\right)}{q^{n}(\boldsymbol{X}, \boldsymbol{Y})} \geq 1\right\}\right] .
$$

Writing the probability as an expectation given $(\boldsymbol{U}, \boldsymbol{X}, \boldsymbol{Y})$ and applying the truncated union bound to the union over $i$, we obtain

$$
\bar{p}_{\mathrm{e}, 0} \leq \mathbb{E}\left[\min \left\{1,\left(M_{0}-1\right) \mathbb{P}\left[\bigcup_{j \neq 1}\left\{\frac{q^{n}\left(\overline{\boldsymbol{X}}^{(j)}, \boldsymbol{Y}\right)}{q^{n}(\boldsymbol{X}, \boldsymbol{Y})} \geq 1\right\} \mid \boldsymbol{U}, \boldsymbol{X}, \boldsymbol{Y}\right]\right\}\right]
$$


Applying the same argument to the union over $j$, we obtain the desired upper bound.

Before proceeding, we introduce some standard notation and terminology associated with the method of types (e.g. see [15, Ch. 2]). For a given joint type, say $\widetilde{P}_{U X}$, the type class $T^{n}\left(\widetilde{P}_{U X}\right)$ is defined to be the set of all sequences in $\mathcal{U}^{n} \times \mathcal{X}^{n}$ with type $\widetilde{P}_{U X}$. Similarly, for a given joint type $\widetilde{P}_{U X}$ and sequence $\boldsymbol{u} \in T^{n}\left(\widetilde{P}_{U}\right)$, the conditional type class $T_{\boldsymbol{u}}^{n}\left(\widetilde{P}_{U X}\right)$ is defined to be the set of all sequences $\boldsymbol{x}$ such that $(\boldsymbol{u}, \boldsymbol{x}) \in T^{n}\left(\widetilde{P}_{U X}\right)$.

In the remainder of the appendix, we consider the constant-composition ensemble, described by

$$
\begin{aligned}
P_{\boldsymbol{U}}(\boldsymbol{u}) & =\frac{1}{\left|T^{n}\left(Q_{U}\right)\right|} \mathbb{1}\left\{\boldsymbol{u} \in T^{n}\left(Q_{U}\right)\right\} \\
P_{\boldsymbol{X} \mid \boldsymbol{U}}(\boldsymbol{x} \mid \boldsymbol{u}) & =\frac{1}{\left|T_{\boldsymbol{u}}^{n}\left(Q_{X \mid U}\right)\right|} \mathbb{1}\left\{\boldsymbol{x} \in T_{\boldsymbol{u}}^{n}\left(Q_{X \mid U}\right)\right\} .
\end{aligned}
$$

Here we have assumed that $Q_{U X}$ is a joint type for notational convenience; more generally, we can approximate $Q_{U X}$ by a joint type and the analysis is unchanged.

\section{B. Derivation of the Primal Expression}

We will derive (6) by showing that the error exponent of $\bar{p}_{\mathrm{e}, 0}$ (i.e. $\lim \inf _{n \rightarrow \infty}-\frac{1}{n} \log \bar{p}_{\mathrm{e}, 0}$ ) is lower bounded by

$$
\begin{aligned}
& E_{\mathrm{r}, 0}^{\mathrm{cc}}\left(Q_{U X}, R_{0}, R_{1}\right) \triangleq \min _{P_{U X Y}: P_{U X}=Q_{U X}} \min _{\substack{\widetilde{P}_{U X Y}: \widetilde{P}_{U X}=Q_{U X}, \widetilde{P}_{Y}=P_{Y} \\
\mathbb{E}_{\tilde{P}}[\log q(X, Y)] \geq \mathbb{E}_{P}[\log q(X, Y)]}} \\
& D\left(P_{U X Y} \| Q_{U X} \times W\right)+\left[I_{\widetilde{P}}(U ; Y)+\left[I_{\widetilde{P}}(X ; Y \mid U)-R_{1}\right]^{+}-R_{0}\right]^{+} .
\end{aligned}
$$

The objective is always positive when $P_{U X Y}$ is bounded away from $Q_{U X} \times W$. Hence, and by applying a standard continuity argument as in [2, Lemma1], we may substitute $P_{U X Y}=Q_{U X} \times W$ to obtain the desired rate condition in (6).

We obtain (65) by analyzing (59) using the method of types. Except where stated otherwise, it should be understood that unions, summations, and minimizations over joint distributions (e.g. $P_{U X Y}$ ) are only over joint types, rather than being over all distributions.

Let us first condition on $\boldsymbol{U}=\boldsymbol{u}, \boldsymbol{X}=\boldsymbol{x}, \boldsymbol{Y}=\boldsymbol{y}$ and $\overline{\boldsymbol{U}}=\overline{\boldsymbol{u}}$ being fixed sequences, and let $P_{U X Y}$ and $\widehat{P}_{U Y}$ respectively denote the joint types of $(\boldsymbol{u}, \boldsymbol{x}, \boldsymbol{y})$ and $(\overline{\boldsymbol{u}}, \boldsymbol{y})$. We can write the inner probability in (59) as

$$
\mathbb{P}\left[\bigcup_{\substack{\widetilde{P}_{U X Y}: \widetilde{P}_{U X}=Q_{U X}, \widetilde{P}_{U Y}=\widehat{P}_{U Y} \\ \mathbb{E}_{\widetilde{P}}[\log q(X, Y)] \geq \mathbb{E}_{P}[\log q(X, Y)]}}\left\{(\overline{\boldsymbol{u}}, \overline{\boldsymbol{X}}, \boldsymbol{y}) \in T^{n}\left(\widetilde{P}_{U X Y}\right)\right\} \mid \overline{\boldsymbol{U}}=\overline{\boldsymbol{u}}\right] .
$$

Note that the constraint $\widetilde{P}_{U X}=Q_{U X}$ arises since every $(\boldsymbol{u}, \boldsymbol{x})$ pair has joint type $Q_{U X}$ by construction. Applying the union bound, the property of types $\mathbb{P}\left[(\overline{\boldsymbol{u}}, \overline{\boldsymbol{X}}, \boldsymbol{y}) \in T^{n}\left(\widetilde{P}_{U X Y}\right) \mid \overline{\boldsymbol{U}}=\overline{\boldsymbol{u}}\right] \leq e^{-n I_{\widetilde{P}}(X ; Y \mid U)}$ [15, Ch. 2], and the fact that the number of joint types is polynomial in $n$, we see that the negative normalized (by $\frac{1}{n}$ ) logarithm of (66) is lower bounded by

$$
\min _{\substack{\widetilde{P}_{U X Y}: \widetilde{P}_{U X}=Q_{U X}, \widetilde{P}_{U Y}=\widehat{P}_{U Y} \\ \mathbb{E}_{\widetilde{P}}[\log q(X, Y)] \geq \mathbb{E}_{P}[\log q(X, Y)]}} I_{\widetilde{P}}(X ; Y \mid U)
$$

plus an asymptotically vanishing term. 
Next, we write the inner expectation in (59) (conditioned on $\boldsymbol{U}=\boldsymbol{u}, \boldsymbol{X}=\boldsymbol{x}$ and $\boldsymbol{Y}=\boldsymbol{y}$ ) as

$$
\begin{aligned}
\sum_{\widehat{P}_{U Y}: \widehat{P}_{U}=Q_{U}, \widehat{P}_{Y}=P_{Y}} \mathbb{P}\left[(\overline{\boldsymbol{U}}, \boldsymbol{y}) \in T^{n}\left(\widehat{P}_{U Y}\right)\right] \min \{1, \\
\left.M_{1} \mathbb{P}[\underbrace{}_{\substack{\widetilde{P}_{U X Y}: \widetilde{P}_{U X}=Q_{U X}, \widetilde{P}_{U Y}=\widehat{P}_{U Y} \\
\mathbb{E}_{\widetilde{P}}[\log q(X, Y)] \geq \mathbb{E}_{P}[\log q(X, Y)]}}\left\{(\overline{\boldsymbol{u}}, \overline{\boldsymbol{X}}, \boldsymbol{y}) \in T^{n}\left(\widetilde{P}_{U X Y}\right)\right\} \mid \overline{\boldsymbol{U}}=\overline{\boldsymbol{u}}]\right\},
\end{aligned}
$$

where now $\overline{\boldsymbol{u}}$ denotes an arbitrary sequence such that $(\boldsymbol{u}, \boldsymbol{y}) \in T^{n}\left(\widehat{P}_{U Y}\right)$. Combining 67) with the property of types $\mathbb{P}\left[(\overline{\boldsymbol{U}}, \boldsymbol{y}) \in T^{n}\left(\widehat{P}_{U Y}\right)\right] \leq e^{-n I_{\hat{P}}(U ; Y)}[15$, Ch. 2], we see that the negative normalized logarithm of (68) is lower bounded by

$$
\min _{\widehat{P}_{U Y}: \widehat{P}_{U}=Q_{U}, \widehat{P}_{Y}=P_{Y}} I_{\widehat{P}}(U ; Y)+\min _{\substack{\widetilde{P}_{U X Y}: \widetilde{P}_{U X}=Q_{U X}, \widetilde{P}_{U Y}=\widehat{P}_{U Y} \\ \mathbb{E}_{\widetilde{P}}[\log q(X, Y)] \geq \mathbb{E}_{P}[\log q(X, Y)]}}\left[I_{\widetilde{P}}(X ; Y \mid U)-R_{1}\right]^{+}
$$

plus an asymptotically vanishing term. Combining the two minimizations into one via the constraint $\widetilde{P}_{U Y}=\widehat{P}_{U Y}$, we see that the right-hand side of (69) coincides with that of (6) (note, however, that $P_{U X Y}$ need not equal $Q_{U X} \times W$ at this stage). Finally, the derivation of (65) is concluded by handling the outer expectation in (59) in the same way as the inner one, applying the property $\mathbb{P}\left[(\boldsymbol{U}, \boldsymbol{X}, \boldsymbol{Y}) \in T^{n}\left(P_{U X Y}\right)\right] \leq e^{-n D\left(P_{U X Y} \| Q_{U X} \times W\right)}$ [15, Ch. 2] (which follows since $(\boldsymbol{U}, \boldsymbol{X})$ is uniform on $\left.T^{n}\left(Q_{U X}\right)\right)$, and expanding the minimization set from joint types to general joint distributions.

\section{Derivation of the Dual Expression}

Expanding (59) and applying Markov's inequality and $\min \{1, \alpha\} \leq \alpha^{\rho}(\rho \in[0,1])$, we obtain

$$
\bar{p}_{e, 0} \leq \sum_{\boldsymbol{u}, \boldsymbol{x}, \boldsymbol{y}} P_{\boldsymbol{U}}(\boldsymbol{u}) P_{\boldsymbol{X} \mid \boldsymbol{U}}(\boldsymbol{x} \mid \boldsymbol{u}) W^{n}(\boldsymbol{y} \mid \boldsymbol{x})\left(M_{0} \sum_{\overline{\boldsymbol{u}}} P_{\boldsymbol{U}}(\overline{\boldsymbol{u}})\left(M_{1} \frac{\sum_{\overline{\boldsymbol{x}}} P_{\boldsymbol{X} \mid \boldsymbol{U}}(\overline{\boldsymbol{x}} \mid \overline{\boldsymbol{u}}) q^{n}(\overline{\boldsymbol{x}}, \boldsymbol{y})^{s}}{q^{n}(\boldsymbol{x}, \boldsymbol{y})^{s}}\right)^{\rho_{1}}\right)^{\rho_{0}} .
$$

for any $\rho_{0} \in[0,1], \rho_{1} \in[0,1]$ and $s \geq 0$. Let $a(u, x)$ be an arbitrary function on $\mathcal{U} \times \mathcal{X}$, and let $a^{n}(\boldsymbol{u}, \boldsymbol{x}) \triangleq$ $\sum_{i=1}^{n} a\left(u_{i}, x_{i}\right)$ be its additive $n$-letter extension. Since $(\boldsymbol{U}, \boldsymbol{X})$ and $(\overline{\boldsymbol{U}}, \overline{\boldsymbol{X}})$ have the same joint type (namely, $\left.Q_{U X}\right)$ by construction, we have $a^{n}(\boldsymbol{u}, \boldsymbol{x})=a^{n}(\overline{\boldsymbol{u}}, \overline{\boldsymbol{x}})$, and hence we can write (71) as

$$
\bar{p}_{e, 0} \leq \sum_{\boldsymbol{u}, \boldsymbol{x}, \boldsymbol{y}} P_{\boldsymbol{U}}(\boldsymbol{u}) P_{\boldsymbol{X} \mid \boldsymbol{U}}(\boldsymbol{x} \mid \boldsymbol{u}) W^{n}(\boldsymbol{y} \mid \boldsymbol{x})\left(M_{0} \sum_{\overline{\boldsymbol{u}}} P_{\boldsymbol{U}}(\overline{\boldsymbol{u}})\left(M_{1} \frac{\sum_{\overline{\boldsymbol{x}}} P_{\boldsymbol{X} \mid \boldsymbol{U}}(\overline{\boldsymbol{x}} \mid \overline{\boldsymbol{u}}) q^{n}(\overline{\boldsymbol{x}}, \boldsymbol{y})^{s} e^{a^{n}(\overline{\boldsymbol{u}}, \overline{\boldsymbol{x}})}}{q^{n}(\boldsymbol{x}, \boldsymbol{y})^{s} e^{a^{n}(\boldsymbol{u}, \boldsymbol{x})}}\right)^{\rho_{1}}\right)^{\rho_{0}}
$$

Upper bounding each constant-composition distribution by a polynomial factor times the corresponding i.i.d. distribution (i.e. $P_{\boldsymbol{U}}(\boldsymbol{u}) \leq(n+1)^{|\mathcal{U}|-1} \prod_{i=1}^{n} Q_{U}\left(u_{i}\right)$ [15, Ch. 2]), we see the exponent of $\bar{p}_{\mathrm{e}, 0}$ is lower bounded by

$$
\max _{\rho_{0} \in[0,1], \rho_{1} \in[0,1]} E_{0}\left(Q_{U X}, \rho_{0}, \rho_{1}\right)-\rho_{0}\left(R_{0}+\rho_{1} R_{1}\right)
$$

where

$$
E_{0}\left(Q_{U X}, \rho_{0}, \rho_{1}\right) \triangleq \sup _{s \geq 0, a(\cdot, \cdot)}-\log \sum_{u, x} Q_{U X}(u, x) W(y \mid x)\left(\sum_{\bar{u}} Q_{U}(\bar{u})\left(\frac{\sum_{\bar{x}} Q_{X \mid U}(\bar{x} \mid \bar{u}) q(\bar{x}, y)^{s} e^{a(\bar{u}, \bar{x})}}{q(x, y)^{s} e^{a(u, x)}}\right)^{\rho_{1}}\right)^{\rho_{0}} .
$$

We obtain (8) in the same way as Gallager's single-user analysis [18, Sec. 5.6] by evaluating the partial derivative of the objective in (73) at $\rho_{0}=0$ (see also [19] for the corresponding approach to deriving the LM rate). 


\section{APPENDIX B}

\section{FURTHER NumERICAL TECHNIQUES USED}

In this section, we present further details of our numerical techniques for the sake of reproducibility. Except where stated otherwise, the implementations were done in C. Our code is available online [14].

The algorithms here do not play a direct role in establishing Counter-Example 1] We thus resort to "ad-hoc" approaches with manually-tuned parameters. In particular, we make no claims regarding the convergence of these algorithms or their effectiveness in handling channels and decoding metrics differing from Counter-Example 1

\section{A. Evaluating $I_{\mathrm{LM}}(Q)$ via the Dual Expression and Gradient Descent}

Here we describe how we optimized the parameters in (4) for a fixed input distribution $Q$ to produce the dual values plotted in Figure 1. Note that replacing the optimization by fixed values leads to a lower bound, whereas for the primal expression it led to an upper bound. Thus, since the two are very close in Figure 1 we can be assured that the true value of $I_{\mathrm{LM}}(Q)$ has been characterized accurately, at least for the values of $Q$ shown. While we focus on the binary-input setting here, the techniques can be applied to an arbitrary mismatched DMC. For brevity, we write $a_{x} \triangleq a(x)$.

Let $Q$ be given, and let $I(\boldsymbol{v})$ be the corresponding objective in (4) as a function of $\boldsymbol{v} \triangleq\left[\begin{array}{lll}s & a_{0} & a_{1}\end{array}\right]^{T}$. Moreover, let $\nabla I(\boldsymbol{v})$ denote the $3 \times 1$ corresponding gradient vector containing the partial derivatives of $I(\cdot)$. These are all easily evaluated in closed form by a direct differentiation. We used the following standard gradient descent algorithm, which depends on the initial values $\left(s^{(0)}, a_{0}^{(0)}, a_{1}^{(0)}\right)$, a sequence of step sizes $\left\{t^{(i)}\right\}$, and a termination parameter $\epsilon$ :

1) Set $i=0$ and initialize $\boldsymbol{v}^{(0)}=\left[s^{(0)} a_{0}^{(0)} a_{1}^{(0)}\right]^{T}$;

2) Set $\boldsymbol{v}^{(i+1)}=\boldsymbol{v}^{(i)}-t^{(i)} \nabla I\left(\boldsymbol{v}^{(i)}\right)$;

3) If $\left\|\nabla I\left(\boldsymbol{v}^{(i+1)}\right)\right\| \leq \epsilon$ then terminate; otherwise, increment $i$ and return to Step 2 .

We used the initial parameters $\left(s^{(0)}, a_{0}^{(0)}, a_{1}^{(0)}\right)=(1,0,0)$, a fixed step size $t^{(i)}=1$, and a termination parameter $\epsilon=10^{-6}$.

Note that we have ignored the constraint $s \geq 0$, but this has no effect on the maximization. This is seen by noting that $s$ is a Lagrange multiplier corresponding to the constraint on the metric in (3), and the inequality therein can be replaced by an equality as long as $I_{\mathrm{LM}}(Q)>0$ [3, Lemma 1]. The equality constraint yields a Lagrange multiplier on $\mathbb{R}$, rather than $\mathbb{R}_{+}$.

\section{B. Evaluating $I_{\mathrm{SC}}^{(2)}\left(Q_{U X}\right)$ via the Dual Expression and Gradient Descent}

To obtain the dual curve for $I_{\mathrm{SC}}^{(2)}$ in Figure 1, we optimized the parameters in (7)- 8 in a similar fashion to the previous subsection. In fact, the optimization of (7) was done exactly as above, with the same initial parameters (i.e. initializing $s=1$ and $a(u, x)=0$ for all $(u, x)$ ). By letting (7) hold with equality, the solution to this optimization gives a value for $R_{1}$.

Handling the optimization in (8) was less straightforward. We were unable to verify the joint concavity of the objective in $\left(\rho_{1}, s, a\right)$, and we in fact found a naive application of gradient descent to be problematic due to overly 
large changes in $\rho_{1}$ on each step. Moreover, while it is safe to ignore the constraint $s \geq 0$ in the same way as the previous subsection, the same is not true of the constraint $\rho_{1} \in[0,1]$. We proceed by presenting a modified algorithm that handles these issues.

Similarly to the previous subsection, we let $\boldsymbol{v}$ be the vector of parameters, let $I_{0}(\boldsymbol{v})$ denote the objective in (8) with $Q_{U X}$ and $R_{1}$ fixed (the latter chosen as the value given by the evaluation of (7)), and let $\nabla I_{0}(\boldsymbol{v})$ be the corresponding gradient vector. Moreover, we define

$$
\Phi\left(\rho_{1}\right) \triangleq \begin{cases}0 & \rho_{1}<0 \\ \rho_{1} & \rho_{1} \in[0,1] \\ 1 & \rho_{1}>1 .\end{cases}
$$

Finally, we let $\boldsymbol{v}_{-\rho_{1}}$ denote the vector $\boldsymbol{v}$ with the entry corresponding to $\rho_{1}$ removed, and similarly for other vectors (e.g. $\left.\left(\nabla I_{0}(\boldsymbol{v})\right)_{-\rho_{1}}\right)$.

We applied the following variation of gradient descent, which depends on the initial parameters, the step sizes $\left\{t^{(i)}\right\}$, and two parameters $\epsilon$ and $\epsilon^{\prime}$ :

1) Set $i=0$ and initialize $\boldsymbol{v}^{(0)}$.

2) Set $\boldsymbol{v}_{-\rho_{1}}^{(i+1)}=\boldsymbol{v}_{-\rho_{1}}^{(i)}-t^{(i)}\left(\nabla I\left(\boldsymbol{v}^{(i)}\right)\right)_{-\rho_{1}}$.

3) If $\left\|\left(\nabla I_{0}\left(\boldsymbol{v}^{(i)}\right)\right)_{-\rho_{1}}\right\| \leq \epsilon^{\prime}$ then set $\rho_{1}^{(i+1)}=\Phi\left(\rho_{1}^{(i)}-\left.t^{(i)} \frac{\partial I_{0}}{\partial \rho_{1}}\right|_{\boldsymbol{v}=\boldsymbol{v}^{(i)}}\right)$; otherwise set $\rho_{1}^{(i+1)}=\rho_{1}^{(i)}$.

4) Terminate if either of the following conditions hold: (i) $\left\|\nabla I\left(\boldsymbol{v}^{(i+1)}\right)\right\| \leq \epsilon$; (ii) $\left\|\left(\nabla I_{0}\left(\boldsymbol{v}^{(i+1)}\right)\right)_{-\rho_{1}}\right\| \leq \epsilon$ and $\rho_{1}^{(i+1)} \in\{0,1\}$. Otherwise, increment $i$ and return to Step 2.

In words, $\rho_{1}$ is only updated if the norm of the gradient corresponding to $(s, a)$ is sufficiently small, and the algorithm may terminate when $\rho_{1}$ saturates to one of the two endpoints of $[0,1]$ (rather than arriving at a local maximum). We initialized $s$ and $\rho_{1}$ to 1 , and each $a(u, x)$ to zero. We again used a constant step size $t^{(i)}=1$, and we chose the parameters $\epsilon=10^{-6}$ and $\epsilon^{\prime}=10^{-2}$.

\section{Evaluating $I_{\mathrm{SC}}^{(2)}\left(Q_{U X}\right)$ via the Primal Expression}

Since we only computed the primal expression for $I_{\mathrm{SC}}^{(2)}\left(Q_{U X}\right)$ with a relatively small number of input distributions (namely, those shown in Figure 1), computational complexity was a minor issue, so we resorted to the generalpurpose software CVX for MATLAB [20]. In the same way as the previous subsection, we solved the right-hand side of (5) to find $R_{1}$, then substituted the resulting value into (6) to find $R_{0}$.

\section{APPENDIX C}

\section{Achievability of (11) via Expurgated Parallel Coding}

Here we outline how the achievable rate of 0.137998 nats/use in (11) can be obtained using Lapidoth's expurgated parallel coding rate. We verified this value by evaluating the primal expressions in [9] using CVX [20], and also by evaluating the equivalent dual expressions in [6] by a suitable adaptation of the dual optimization parameters 
for superposition coding given in Section [II-D We focus our attention on the latter, since it immediately provides a concrete lower bound even when the optimization parameters are slightly suboptimal.

The parameters to Lapidoth's rate are two finite alphabets $\mathcal{X}_{1}$ and $\mathcal{X}_{2}$, two corresponding input distributions $Q_{1}$ and $Q_{2}$, and a function $\phi\left(x_{1}, x_{2}\right)$ mapping $\mathcal{X}_{1}$ and $\mathcal{X}_{2}$ to the channel input alphabet. For any such parameters, the rate $R=R_{1}+R_{2}$ is achievable provided that [6], [8]

$$
\begin{aligned}
& R_{1} \leq \sup _{s \geq 0, a(\cdot, \cdot)} \mathbb{E}\left[\log \frac{q\left(\phi\left(X_{1}, X_{2}\right), Y\right)^{s} e^{a\left(X_{1}, X_{2}\right)}}{\mathbb{E}\left[q\left(\phi\left(\bar{X}_{1}, X_{2}\right), Y\right)^{s} e^{a\left(\bar{X}_{1}, X_{2}\right)} \mid X_{2}, Y\right]}\right] \\
& R_{2} \leq \sup _{s \geq 0, a(\cdot, \cdot)} \mathbb{E}\left[\log \frac{q\left(\phi\left(X_{1}, X_{2}\right), Y\right)^{s} e^{a\left(X_{1}, X_{2}\right)}}{\mathbb{E}\left[q\left(\phi\left(X_{1}, \bar{X}_{2}\right), Y\right)^{s} e^{a\left(X_{1}, \bar{X}_{2}\right)} \mid X_{1}, Y\right]}\right],
\end{aligned}
$$

and at least one of the following holds:

$$
\begin{aligned}
& R_{1} \leq \sup _{\rho_{2} \in[0,1], s \geq 0, a(\cdot, \cdot)} \mathbb{E}\left[\log \frac{\left(q\left(\phi\left(X_{1}, X_{2}\right), Y\right)^{s} e^{a\left(X_{1}, X_{2}\right)}\right)^{\rho_{2}}}{\mathbb{E}\left[\left(\mathbb{E}\left[q\left(\phi\left(\bar{X}_{1}, \bar{X}_{2}\right), Y\right)^{s} e^{a\left(\bar{X}_{1}, \bar{X}_{2}\right)} \mid \bar{X}_{1}\right]\right)^{\rho_{2}} \mid Y\right]}\right]-\rho_{2} R_{2} \\
& R_{2} \leq \sup _{\rho_{1} \in[0,1], s \geq 0, a(\cdot, \cdot)} \mathbb{E}\left[\log \frac{\left(q\left(\phi\left(X_{1}, X_{2}\right), Y\right)^{s} e^{a\left(X_{1}, X_{2}\right)}\right)^{\rho_{1}}}{\mathbb{E}\left[\left(\mathbb{E}\left[q\left(\phi\left(\bar{X}_{1}, \bar{X}_{2}\right), Y\right)^{s} e^{a\left(\bar{X}_{1}, \bar{X}_{2}\right)} \mid \bar{X}_{2}\right]\right)^{\rho_{1}} \mid Y\right]}\right]-\rho_{1} R_{1},
\end{aligned}
$$

where $\left(X_{1}, X_{2}, Y, \bar{X}_{1}, \bar{X}_{2}\right) \sim Q_{1}\left(x_{1}\right) Q_{2}\left(x_{2}\right) W\left(y \mid \phi\left(x_{1}, x_{2}\right)\right) Q_{1}\left(\bar{x}_{1}\right) Q_{2}\left(\bar{x}_{2}\right)$.

Recall the input distribution $Q_{U X}$ for superposition coding on the second-order product channel given in (47)-(49). Denoting the four inputs of the product channel as $\{(0,0),(0,1),(1,0),(1,1)\}$, we set $\mathcal{X}_{1}=\{(0,0),(0,1),(1,0)\}$, $\mathcal{X}_{2}=\mathcal{U}=\{0,1\}$, and

$$
\begin{aligned}
Q_{X_{1}} & =\frac{1}{1-Q_{1}^{2}}\left[\begin{array}{lll}
Q_{0}^{2} & Q_{0} Q_{1} & Q_{0} Q_{1}
\end{array}\right] \\
Q_{X_{2}} & =\left[\begin{array}{ll}
1-Q_{1}^{2} & Q_{1}^{2}
\end{array}\right] \\
\phi\left(x_{1}, x_{2}\right) & = \begin{cases}x_{1} & x_{2}=0 \\
(1,1) & x_{2}=1 .\end{cases}
\end{aligned}
$$

This induces a joint distribution $Q_{X_{1} X_{2} X}\left(x_{1}, x_{2}, x\right)=Q_{X_{1}}\left(x_{1}\right) Q_{X_{2}}\left(x_{2}\right) \mathbb{1}\left\{x=\phi\left(x_{1}, x_{2}\right)\right\}$. The idea behind this choice is that the marginal distribution $Q_{X_{2} X}$ coincides with our choice of $Q_{U X}$ for SC.

By the structure of our input distributions, there is in fact a one-to-one correspondence between $(u, x)$ and $\left(x_{1}, x_{2}\right)$, thus allowing us to immediately use the dual parameters $\left(s, a, \rho_{1}\right)$ from SC for the expurgated parallel coding rate. More precisely, using the superscripts $(\cdot)^{\mathrm{sc}}$ and $(\cdot)^{\mathrm{ex}}$ to distinguish between the two ensembles, we set

$$
\begin{aligned}
R_{1}^{\mathrm{ex}} & =R_{1}^{\mathrm{sc}} \\
R_{2}^{\mathrm{ex}} & =R_{0}^{\mathrm{sc}} \\
s^{\mathrm{ex}} & =s^{\mathrm{sc}} \\
a^{\mathrm{ex}}\left(x_{1}, x_{2}\right) & =a^{\mathrm{sc}}\left(x_{2}, \phi\left(x_{1}, x_{2}\right)\right) \\
\rho_{1}^{\mathrm{ex}} & =\rho_{1}^{\mathrm{sc}} .
\end{aligned}
$$


Using these identifications along with the choices of the superposition coding parameters in (52)-(56), we verified numerically that the right-hand side of (75) (respectively, (78) ) coincides with that of (7) (respectively, (8)). Finally, to conclude that the expurgated parallel coding rate recovers (11), we numerically verified that the rate $R_{2}$ resulting from (75) and (78) (which, from (51), is 0.0356005) also satisfies (76). In fact, the inequality is strict, with the right-hand side of (76) being at least 0.088 .

\section{REFERENCES}

[1] J. Hui, "Fundamental issues of multiple accessing," Ph.D. dissertation, MIT, 1983.

[2] I. Csiszár and J. Körner, "Graph decomposition: A new key to coding theorems," IEEE Trans. Inf. Theory, vol. 27, no. 1, pp. 5-12, Jan. 1981.

[3] I. Csiszár and P. Narayan, "Channel capacity for a given decoding metric," IEEE Trans. Inf. Theory, vol. 45, no. 1, pp. 35-43, Jan. 1995.

[4] N. Merhav, G. Kaplan, A. Lapidoth, and S. Shamai, "On information rates for mismatched decoders," IEEE Trans. Inf. Theory, vol. 40, no. 6, pp. 1953-1967, Nov. 1994.

[5] V. Balakirsky, "Coding theorem for discrete memoryless channels with given decision rule," in Algebraic Coding. Springer Berlin / Heidelberg, 1992, vol. 573, pp. 142-150.

[6] J. Scarlett, A. Martinez, and A. Guillén i Fàbregas, "Multiuser coding techniques for mismatched decoding," 2013, submitted to IEEE Trans. Inf. Theory [Online: http://arxiv.org/abs/1311.6635].

[7] A. Somekh-Baruch, "On achievable rates and error exponents for channels with mismatched decoding," IEEE Trans. Inf. Theory, vol. 61, no. 2, pp. 727-740, Feb. 2015.

[8] J. Scarlett, "Reliable communication under mismatched decoding," Ph.D. dissertation, University of Cambridge, 2014, [Online: http://itc.upf.edu/biblio/1061].

[9] A. Lapidoth, "Mismatched decoding and the multiple-access channel," IEEE Trans. Inf. Theory, vol. 42, no. 5, pp. 1439-1452, Sept. 1996.

[10] A. Ganti, A. Lapidoth, and E. Telatar, "Mismatched decoding revisited: General alphabets, channels with memory, and the wide-band limit," IEEE Trans. Inf. Theory, vol. 46, no. 7, pp. 2315-2328, Nov. 2000.

[11] A. Somekh-Baruch, "A general formula for the mismatch capacity," http://arxiv.org/abs/1309.7964.

[12] S. Verdú and T. S. Han, "A general formula for channel capacity," IEEE Trans. Inf. Theory, vol. 40, no. 4, pp. 1147-1157, July 1994.

[13] V. Balakirsky, "A converse coding theorem for mismatched decoding at the output of binary-input memoryless channels," IEEE Trans. Inf. Theory, vol. 41, no. 6, pp. 1889-1902, Nov. 1995.

[14] J. Scarlett, A. Somekh-Baruch, A. Martinez, and A. Guillén i Fàbregas, "C, Matlab and Mathematica code for 'A counter-example to the mismatched decoding converse for binary-input discrete memoryless channels', ' http://itc.upf.edu/biblio/1076.

[15] I. Csiszár and J. Körner, Information Theory: Coding Theorems for Discrete Memoryless Systems, 2nd ed. Cambridge University Press, 2011.

[16] S. Boyd and L. Vandenberghe, Convex Optimization. Cambridge University Press, 2004.

[17] Wolfram Mathematica arbitrary-precision numbers. [Online]. Available: $\{$ http://reference.wolfram.com/language/tutorial/ $\}\{$ ArbitraryPrecisionNumbers.html\}

[18] R. Gallager, Information Theory and Reliable Communication. John Wiley \& Sons, 1968.

[19] S. Shamai and I. Sason, "Variations on the Gallager bounds, connections, and applications," IEEE Trans. Inf. Theory, vol. 48, no. 12, pp. 3029-3051, Dec. 2002.

[20] M. Grant and S. Boyd, "CVX: Matlab software for disciplined convex programming," http://cvxr.com/cvx. 